\title{
Utilization of Image Intensifiers in Astronomy
}

\author{
S. Vítek, K. Fliegel, P. Páta, P. Koten
}

\begin{abstract}
In this paper we present the properties of image intensifiers, used together with fast TV cameras for astronomical purposes within the MAIA project(Meteor Automatic Imager and Analyser, primarily focused on observing meteoric events with high time resolution). The main objective of our paper is to evaluate the suitability of these devices for astronomical purposes in terms of noise, temporal and spectral analysis.
\end{abstract}

Keywords: image intensifier, astronomy, meteors.

\section{Introduction}

An interesting technique (that has became relatively inexpensive in recent years) for increasing the time resolution of any astronomical instrument is the use of a modern CCD camera with a fast framerate of 50 or more frames per second. This type of system has a significant deficiency in terms of reduced sensitivity; the solution may to use an image intensifier. This paper describes our experience with a device of this kind.

The MAIA astronomical instrument [2] uses a second generation Mullard XX1332 image intensifier. The tube assembly of this $50 / 40 \mathrm{~mm}$ inverter (typical gain 30000 to $60000 \mathrm{~lm} / \mathrm{lm}$, resolution $30 \mathrm{lp} / \mathrm{mm}$ ) is designed to be incorporated in night vision devices, in particular in tank driving periscopes. This leads to some properties which significantly define the lim- its and the possibilities of using a device of this kind in astronomy.

\section{Electrooptical characteristics}

The instrument is equipped with an input aperture lens (Pentax SMC FA 1.4/50 mm) and an inner camera lens (Pentax H1214-M 1.4/12 mm) [4]. The spectral transparency of the two lenses (input and camera), the spectral sensitivity of the image intensifier, the spectral sensitivity of the camera, the spectrum of the light at the output of the image intensifier, the spatial resolution of the input lens and the image intensifier, and also the spatial resolution of the whole system are among the most important parameters tested and presented in this paper.

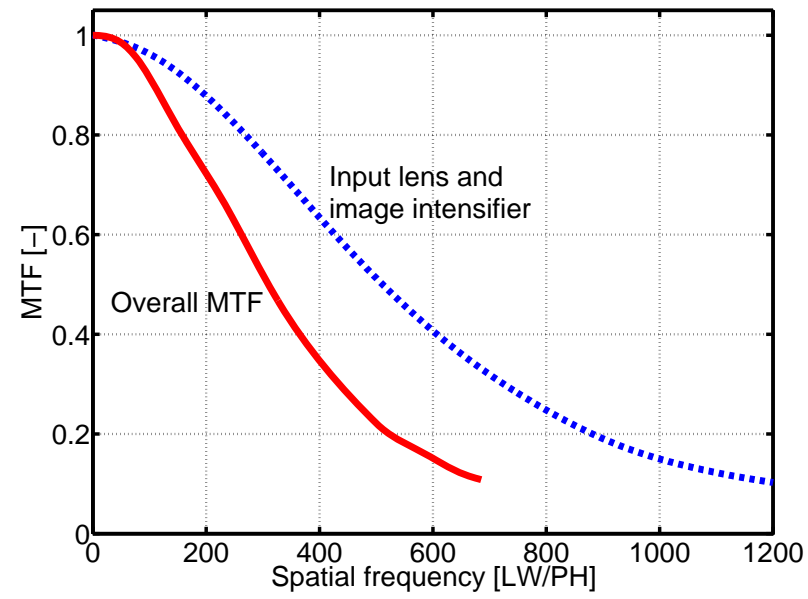

(b)

Fig. 1: The normalized gain of the system (measured at $650 \mathrm{~nm}$ ) describes the automatic gain control as a nonlinearity in the image intensifier (a), the overall MTF of the system, including the camera (solid line) and the partial MTF of the image intensifier with the lens (dashed line) (b) 


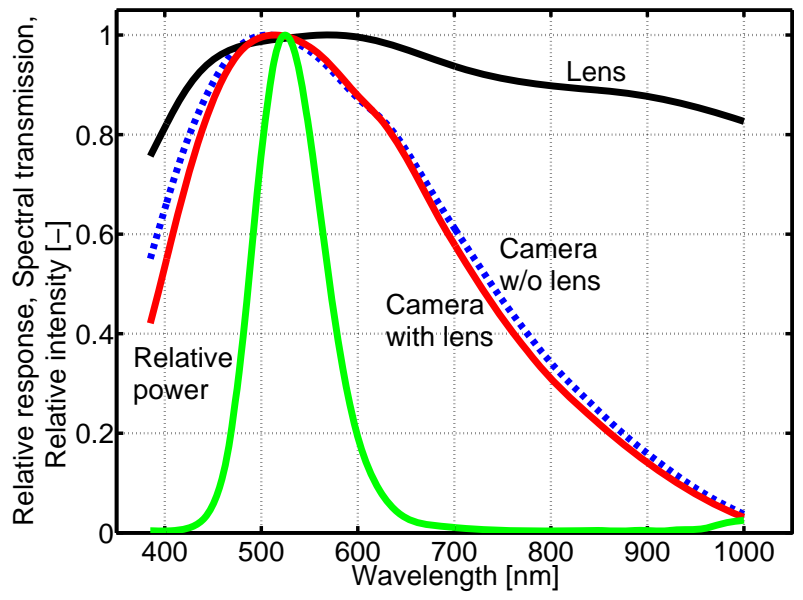

(a)

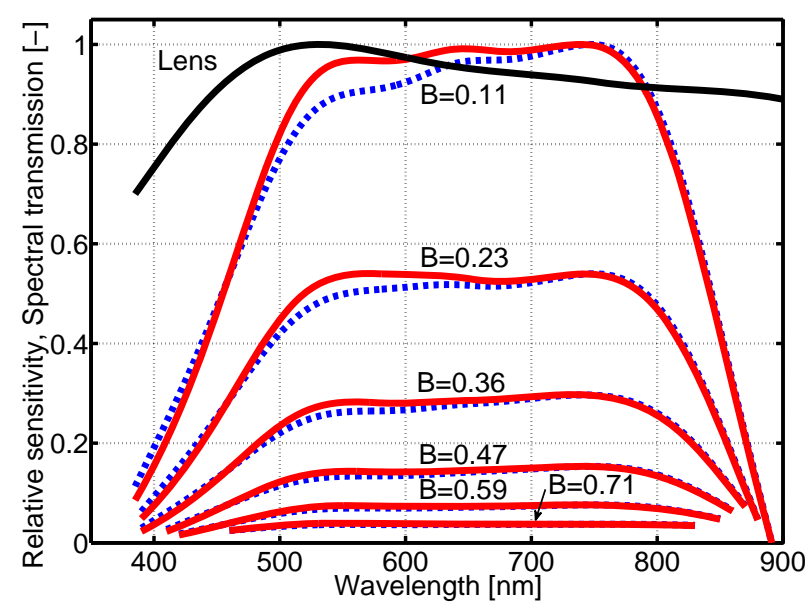

(b)

Fig. 2: Relative spectral response of the camera with (or without) a lens, and the relative power of the light at the output screen of the image intensifier (a), relative overall spectral sensitivity of the system for different digital levels B in the output image $(B=1$ white, $B=0$ black) $(\mathrm{b})$

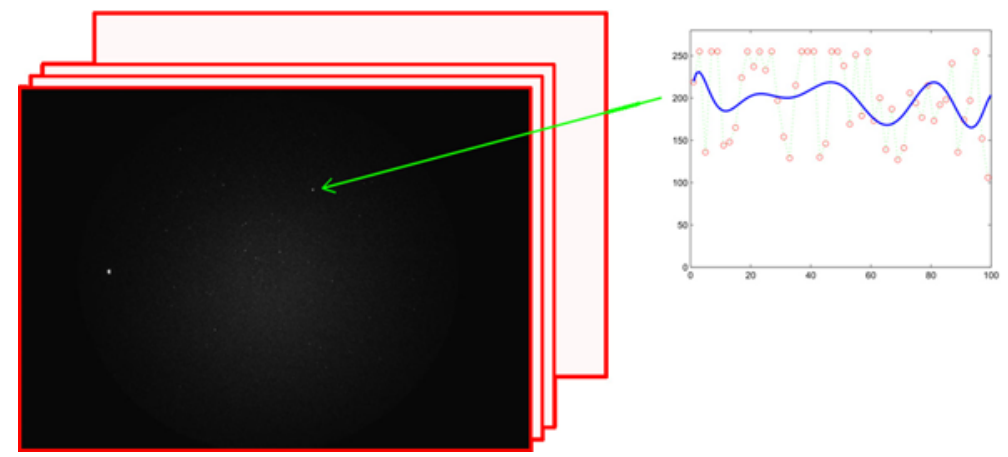

Fig. 3: Temporal changes in stellar object flux

\section{$2.1 \quad$ Spectral response}

The spectral response was measured independently for all parts of the system [3]. The experimental setup consisted of the LOT-Oriel collimated halogen light source, the LOT-Oriel Omni 150 computer controlled monochromator, the expander to get even illumination of the image sensor, and the Avantes AvaSpec3648 fiber optic spectrometer. The measurement results are shown in Figure 2.

\subsection{Spatial resolution}

The MTF was measured using a test chart according to ISO 12233. This chart can be used to evaluate MTF with two different approaches, utilizing a slanted edge (an approx. $5^{\circ}$ slightly slanted black bar used to measure the horizontal or vertical spatial frequency response), or a line square wave sweep with the spatial frequency range 100-1000 LW/PH (line widths per picture height). In our case, slanted edges were used to determine the spatial frequency response - see Figure 1(b).

\section{Noise performance}

The noise analysis based on the acquisition of testing video sequences in various light conditions is described in [5]. We choose the Generalized Laplacian Model (GLM) for heavy-tailed noise.

\section{Temporal analysis}

Figure 3 shows the temporal changes in stellar object flux over 100 frames of videosequence. The changes are mainly due to the automatic gain control. This fact puts greater demands on image calibration. We have proposed adaptive flat-fielding for any light conditions.

\section{Conslusions}

The biggest disadvantage of the image intensifier described here is the built-in automatic gain control. As is shown Figure 1(a), the gain decreases rapidly with increasing input power, i.e. if any bright stellar object appears in the field of view. However the image 
intensifier is not a bottleneck of the MAIA device all measured parameters of an image intensifier are far better than the parameters of the CCD camera used for the project.

\section{Acknowledgement}

This work has been supported by grant No. 205/09/1302 Study of sporadic meteors and weak meteor showers using automatic video intensifier cameras of the Grant Agency of the Czech Republic. We would also like to acknowledge grant No. 102/09/0997 from Grant Agency of the Czech Republic.

\section{References}

[1] Koten, P.: Software for processing of meteor video records. Proceedings of the Asteroids, Comets, Meteors 2002 conference, 197, 2002.

[2] Vítek, S., Koten, P., Páta, P., Fliegel, K.: Double-Station Automatic Video Observation of the Meteors. Advances in Astronomy, 2010 (Article ID 943145), 4 pages (2010).
[3] Fliegel, K., Havlín, J.: Imaging photometer with a non-professional digital camera. Proc. SPIE 7 443, 74431Q, 2009.

[4] Fliegel, K., Švihlík, P., Páta, P., Vítek, S., Koten, P.: Meteor automatic imager and analyzer: current status and preprocessing of image data. Applications of Digital Image Processing XXXIV, Proc. SPIE, 2011.

[5] Švihlík, P., Fliegel, K., Koten, P., Vítek, S., Páta, P.: Noise Analysis of MAIA System and Possible Noise Suppressio. Radioengineering, Vol. 20, pp. 110-117, 2011.

Stanislav Vítek

Karel Fliegel

Petr Páta

Faculty of Electrical Engineering

Czech Technical University

Pavel Koten

Astronomical Institute of the

Academy of Sciences of the Czech Republic 